\title{
In vivo quantification of rolling and adhered leukocytes in human sepsis
}

Bjorn K. Fabian-Jessing ${ }^{1,2}$, Michael J. Massey ${ }^{1}$, Michael R. Filbin³ ${ }^{3}$ Peter C. Hou ${ }^{4}$, Henry E. Wang ${ }^{5}$, Hans Kirkegaard ${ }^{2}$, Donald M. Yealy ${ }^{6}$, William C. Aird ${ }^{7}$, John A. Kellum ${ }^{8}$, Derek C. Angus ${ }^{8}$, Nathan I. Shapiro ${ }^{1 *}$ (i) and on behalf of the ProCESS Investigators

\begin{abstract}
Background: The use of in vivo videomicroscopy at the bedside has demonstrated microcirculatory flow disturbances in sepsis. The ability of in vivo videomicroscopy to detect changes in the prevalence of rolling and adhered leukocytes that occur in sepsis is not well-described in humans. We sought to (1) develop methodology for accessing and quantifying sublingual leukocyte rolling and adherence with sidestream dark field (SDF) imaging; (2) compare the number of rolling and adhered leukocytes between patients with septic shock and non-infected controls; and (3) compare the number of rolling and adhered leukocytes between survivors and non-survivors of septic shock.
\end{abstract}

Methods: We included adult (age > 18 years) patients in the emergency department presenting with septic shock prospectively enrolled in the ProCESS trial. We recruited comparison non-infected patients as emergency department controls. Using a SDF videomicroscope, we obtained image sequences from the sublingual mucosa, quantifying rolling and adhered leukocytes per $1 \mathrm{~mm} \times 1 \mathrm{~mm}$ visual field in a standardized 3-s clip. We report data as median and interquartile range and depicted as box plots. We compared groups using the Mann-Whitney $U$ test, considering a $p$ value $<0.05$ significant.

Results: We included a total of 64 patients with septic shock and 32 non-infected controls. The median number of adhered leukocytes per field in the sepsis group was 1.0 (IQR 0-3.5) compared to 0 (0-0) in the non-infected group ( $p<0.001)$. The median number of rolling leukocytes was 26 (10.3-42) in the sepsis group and 9.8 (4.8-17.3) in the non-infected group $(p<0.001)$ per field. Among the patients with sepsis $(n=64)$, there was an increased number of adhered leukocytes in non-survivors compared to survivors (3.0 (1-5.5) vs. $1.0(0-3.0))(p<0.05)$; however, there was no difference in rolling leukocytes (35 (20-48) vs. $26(10-41))(p=0.31)$.

Conclusions: Our results demonstrated a higher number of rolling and adhered leukocytes in patients with septic shock when compared to non-infected controls, and an increased number of adhered leukocytes in non-survivors.

Trial registration: ClinicalTrials.gov, NCT00793442; Registered on 19 November 2008

PG0GM076659 (US NIH Grant/Contract). First submitted 18 July 2007. First posted 2 August 2007.

Keywords: Sepsis, Septic shock, Microcirculation, Leukocyte, Sidestream dark field, SDF

\footnotetext{
* Correspondence: nshapiro@bidmc.harvard.edu

1 Department of Emergency Medicine, Beth Israel Deaconess Medical Center,

Harvard Medical School, 1 Deaconess Road, CC2-W, Boston, MA 02215, USA

Full list of author information is available at the end of the article
}

(c) The Author(s). 2018 Open Access This article is distributed under the terms of the Creative Commons Attribution 4.0 International License (http://creativecommons.org/licenses/by/4.0/), which permits unrestricted use, distribution, and reproduction in any medium, provided you give appropriate credit to the original author(s) and the source, provide a link to the Creative Commons license, and indicate if changes were made. The Creative Commons Public Domain Dedication waiver (http://creativecommons.org/publicdomain/zero/1.0/) applies to the data made available in this article, unless otherwise stated. 


\section{Background}

Endothelial cell dysfunction and microcirculatory flow alterations are elements of sepsis pathophysiology. As a result of the host immune response to infection, there is up-regulation of endothelial and leukocyte adhesion molecules, which initiates leukocyte recruitment and activation [1]. Eventually, adhesive bindings will overcome post-capillary shear stress forces resulting in slowing and rolling, tethering, firm adherence and, ultimately, transmigration (extravasation) of leukocytes [2].

In the experimental setting, many have observed leukocyte-endothelial cell interactions and microcirculatory flow disturbances in sepsis [3, 4], primarily using intravital microscopy in different animal models. Similarly, others using the same tool have observed increased numbers of rolling and adhered leukocytes on the endothelial surface of different organs during experimental sepsis [3, 5]. However, intravital microscopy (as it requires transillumination of the organ of interest and fluorescent dyes) is not readily suitable for human studies. Recent advances in imaging techniques such as sidestream dark field (SDF) imaging have enabled direct in vivo bedside visualization of the human sublingual microcirculation. Multiple SDF studies show a reduction in the capillary density of the microvasculature and a decrease in the adequacy of erythrocyte flow are associated with adverse outcomes in critical illnesses, especially sepsis $[6,7]$. Furthermore, microcirculatory disturbances may occur in the presence of normalized macrocirculation (and macrocirculatory disturbances may occur with intact microcirculation), and microcirculatory changes are independently prognostic of adverse outcomes $[8,9]$.

Since the emphasis of human in vivo studies of the (sublingual) microcirculation has traditionally focused on erythrocyte flow, direct assessment of leukocyteendothelium interactions in humans is limited [10-12]. Based on the experimental theories and initial human studies, we sought to advance the methodology and study leukocyte rolling and adhesion in sepsis. Accordingly, we aimed (1) to develop methodology for accessing and quantifying sublingual leukocyte activation with SDF imaging; (2) to compare the number of rolling and adhered leukocytes between patients with septic shock and non-infected control patients; and (3) to compare the number of rolling and adhered leukocytes between survivors and non-survivors of septic shock.

\section{Methods}

\section{Study design and setting}

This was a prospective, observational, multicenter study comparing SDF images from emergency department (ED) patients with septic shock enrolled in the Protocolized Care for Early Septic Shock (ProCESS) trial [13] to those from non-infected ED control patients. The ED "control" patients presented with non-infectious, nonshock-related conditions. Patient SDF images (see below for details) within $6 \mathrm{~h}$ of enrollment in the ProCESS study protocol were then processed and analyzed offline. The institutional review boards of the University of Pittsburgh, the Beth Israel Deaconess Medical Center, and the participating recruitment sites approved the design. All subjects or their legally authorized representatives provided written informed consent.

\section{Participants \\ Septic shock cohort}

We included adult subjects (age $>18$ years) enrolled in this ancillary study of the ProCESS trial [13]. In brief, we enrolled ED patients within $2 \mathrm{~h}$ of meeting criteria for septic shock ((1) suspected infection, (2) meeting $\geq 2$ criteria for systemic inflammatory response syndrome (SIRS) [14], and (3) evidence of hypoperfusion defined as refractory hypotension (systolic blood pressure < $90 \mathrm{mmHg}$ or required vasopressor use after a minimum $1 \mathrm{~L}$ fluid challenge) or serum lactate $\geq 4 \mathrm{mmol} / \mathrm{L}$.) For this ancillary study, we enrolled at six different participating centers.

\section{Non-infected control cohort}

Control patients were a convenience sample of patients presenting to one of three EDs and having non-infectious complaints that did not meet SIRS criteria and did not have clinical shock.

\section{SDF image acquisition and selection}

The SDF videomicroscope (MicroScan, MicroVision Medical BV, Amsterdam, The Netherlands) is a Food and Drug Administration (FDA)-exempt device, which consists of miniature microscope optics surrounded by light-emitting diodes (LEDs). The LEDs emit a stroboscopic green light with a wavelength of $530 \mathrm{~nm}$, which is absorbed by hemoglobin in red blood cells, and transmitted by leukocytes, which may appear slightly brighter than the illuminated background tissue. SDF imaging allows direct bedside visualization of the sublingual microcirculation. Video images were saved on a laptop computer and uploaded to a server in a de-identified secure fashion.

Using the international consensus criteria [15] and according to the "Microcirculation Image Quality Score" [16], we chose videos for further analysis. We assessed image quality using six factors: illumination (exposure, brightness and contrast), focus (such that most of the vessels appear in the plane of focus), duration (at least $3 \mathrm{~s}$ ), content (absence of bubbles, saliva, or looped vessels), stability (so that the video can be stabilized effectively without blurring or too much net motion), and pressure (no stopped flow in medium/large vessels or 
other indicators of iatrogenic pressure). Scores were 0 (good), 1 (acceptable), or 10 (unacceptable). For the assessment of microcirculatory flow, we analyzed further videos with scores < 10 ; for the quantification of activated leukocytes, we required a quality score $\leq 1$ because we found that it was impossible to identify leukocytes in even slightly out-of-focus videos. Up to three videos per patient were selected for further analysis. Videos analysis was performed by investigators unaware of clinical information and not involved in the clinical study protocol or in image acquisition.

\section{SDF image processing}

We used a hybrid method involving video preprocessing and stabilization in MATLAB (Mathworks, Natick, MA, USA), followed by tracing and semi-quantitative flow analysis in a dedicated software package (Automated Vascular Analysis (AVA), MicroVision Medical BV, Amsterdam, The Netherlands). Pre-processing and stabilization involved the following steps: (1) a $3 \times 3$ median filter is applied to each frame to remove salt-and-pepper noise while preserving relevant image data; (2) inter-frame motion estimate and stabilization using a multiple key-point-feature-based method [17], operating on vessel probability density images [18]; and (3) finally, we enhanced the stabilized images using adaptive histogram equalization [19], then imported pre-processed, stabilized videos into AVA for semiquantitative analysis.

\section{Technique for quantification of adhered and rolling leukocytes}

For leukocyte quantification, we frame averaged preprocessed and stabilized videos using approximately 1.5 frame Gaussian filter kernel widths. This technique blurs out the column of erythrocytes, plasma gaps and non-activated leukocytes, which constitutes the main blood stream in the SDF images, thus making it easier to identify rolling or adhered leukocytes. The frame-averaged videos were clipped to 90 frames (3 s), so that the middle 90 frames of all videos were selected automatically. Rolling and adhered leukocytes were quantified manually with the assistance of the free software program ImageJ (Rasband, W.S., ImageJ, U. S. National Institutes of Health, Bethesda, MD, USA, http://imagej.nih.gov/ij/, 1997-2014), which allowed playback at different speeds.

Leukocytes appear as round "defects" in the erythrocyte column, slightly brighter than the background tissue and plasma gaps. In addition, activated (rolling) leukocytes display a characteristic moving pattern along the edges of the erythrocyte column, closer to the surface of the endothelium. Rolling leukocytes were defined as leukocytes moving slower than the main bloodstream. Adhered leukocytes were defined as leukocytes firmly adhered to the vessel lumen such that they were not moving throughout the 3-s clip (Fig. 1 and Additional file 1). Adhered leukocytes that detached from the lumen during the 3-s observation period were counted as rolling. Videos were analyzed consecutively according to a list with videos in a computer-generated random order labeled with computer-generated random numbers. We counted leukocytes in the entire field of view (FOV), in all vessels. To avoid double-counting, all rolling/ adhered leukocytes were marked manually in a still picture of the FOV in the respective videos to keep track of the leukocytes during playback. To account for unequal density and distribution of in-focus vessels between videos, we extracted vessel lengths of all in-focus vessels, excluding any out-of-focus vessels. Thus, we calculate the number of activated leukocytes in the FOV and per unit vessel length (number of leukocytes $/ \mathrm{mm}$ ), which is independent of the total vessel density.

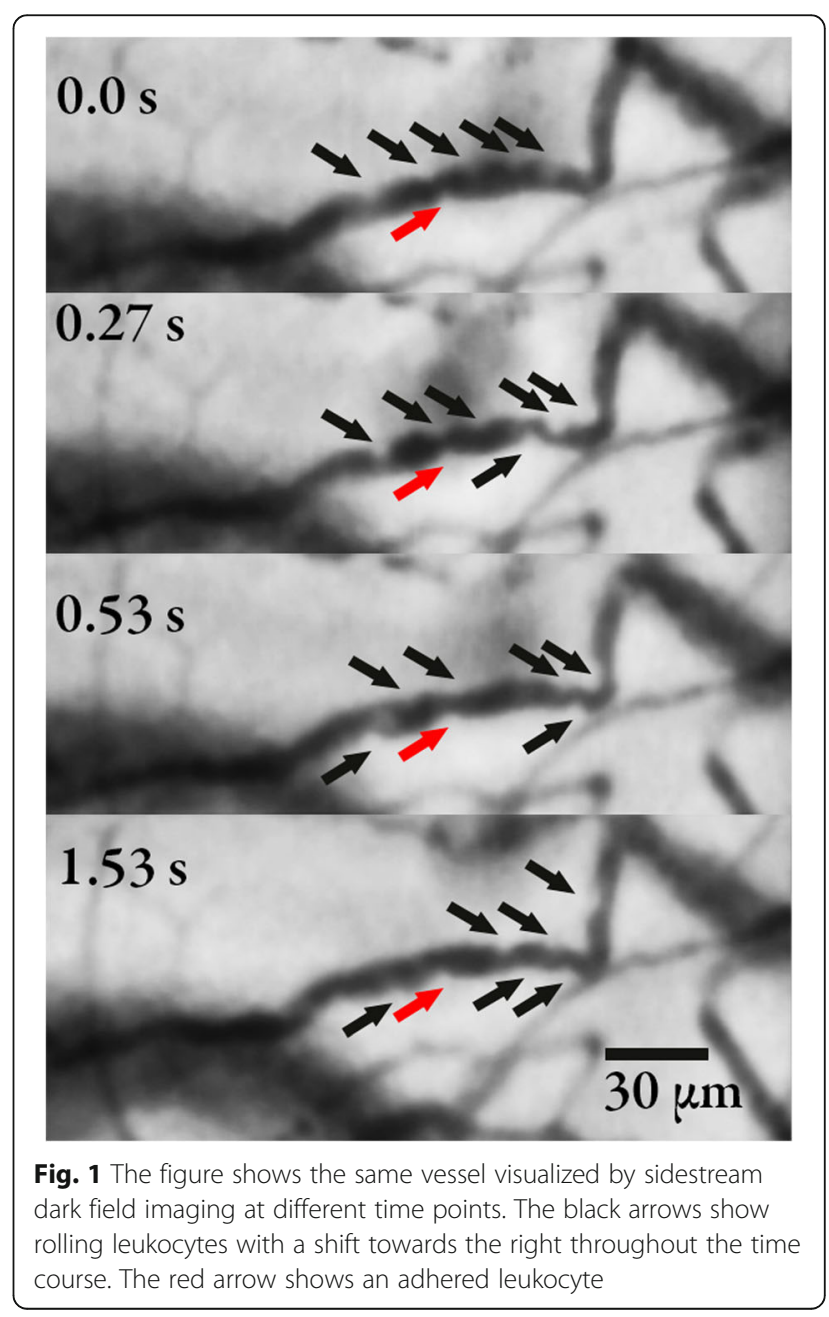




\section{Statistical analysis}

Due to the non-normal distribution of the data we report data as median and interquartile range (IQR) and depict data as box plots. We compared groups using the Mann-Whitney $\mathrm{U}$ test, considering a $p$ value $<0.05$ significant.

\section{Results}

We included 64 patients from the ED presenting with septic shock, who were enrolled in the prospective clinical ProCESS trial, and 32 non-infected control ED patients (Table 1).

\section{Leukocyte rolling and adhesion in sepsis versus control}

There was an increase in the median number of adhered leukocytes per field in the sepsis group 1.0 (IQR 0-3.5) compared to $0(0-0)$ in the non-infected group $(p<0.001)$ (Fig. 2). This corresponded to a median number of adhered leukocytes per unit vessel length of $0.07 / \mathrm{mm}(0-$ $0.23)$ and $0.03 / \mathrm{mm}(0-0)$ for sepsis and non-infected groups, respectively $(p<0.001)$. For the rolling leukocytes, we observed a median of $26(10.3-42)$ in the sepsis group and $9.8(4.8-17.3)$ in the non-infected group $(p<0.001)$ per field (Fig. 3). This corresponded to a median number of rolling leukocytes per unit vessel length of $1.7(0.7-2.7)$ and $0.6 / \mathrm{mm}(0.4-1.0)$, respectively $(p<0.001)$. We also performed a sensitivity analysis by adjusting by perfused vascular density (PVD) and total vascular density (TVD), and found no meaningful differences in the results.

\section{Leukocyte rolling and adhesion in sepsis survivors versus non-survivors}

Among the patients with sepsis $(n=64)$, there was an increase in the number of adhered leukocytes in non-survivors compared to survivors (3.0 (1-5.5) vs. 1.0 $(0-3.0), \quad p<0.05) \quad$ (Fig. 4); this corresponds to a vessel-length adjusted value of $0.17(0.07-0.31)$ vs. 0.07 $(0-0.22), p=0.07$. However, there was no difference in rolling leukocytes $(35(20-48)$ vs. $26(10-41), p=0.31)$; vessel-length adjusted value of 2.0 (1.2-4.1) vs. 1.7 (0.62.5), $p=0.35$ (Fig. 5). Here, we also performed a sensitivity analysis by adjusting by PVD and TVD, and found no meaningful differences in the results.

\section{Discussion}

Our findings are initial evidence in support of the use of the sublingual mucosa as a window for in vivo human studies of leukocyte activation in sepsis as sublingual leukocyte rolling and adherence may hold potential as a diagnostic and prognostic marker. These data provide support for further investigation in this domain.

In prior studies with orthogonal polarization spectral (OPS) and SDF imaging of the human sublingual mucosa rolling leukocytes were generally defined as leukocytes
Table 1 Characteristics of the patients

\begin{tabular}{|c|c|c|}
\hline Characteristic & $\begin{array}{l}\text { Sepsis } \\
(n=64)\end{array}$ & $\begin{array}{l}\text { Control } \\
(n=32)\end{array}$ \\
\hline \multicolumn{3}{|l|}{ Past medical history, $n(\%)$} \\
\hline - Diabetes mellitus & $21(32.8)$ & $6(18.8)$ \\
\hline - Chronic obstructive pulmonary disease & $20(31.3)$ & $2(6.3)$ \\
\hline - Liver disease & $4(6.3)$ & $2(6.3)$ \\
\hline - End-stage renal disease & $4(6.3)$ & $2(6.3)$ \\
\hline - AIDS & $0(0)$ & $2(6.3)$ \\
\hline - Cancer & $19(29.7)$ & $5(15.6)$ \\
\hline Metastatic cancer & $13(20.3)$ & $2(6.3)$ \\
\hline$-\mathrm{CHF}$ & $14(21.9)$ & $2(6.3)$ \\
\hline - Myocardial infarction & $7(10.9)$ & $4(12.5)$ \\
\hline - Dementia & $3(4.7)$ & $1(3.1)$ \\
\hline \multicolumn{3}{|l|}{ Race } \\
\hline - White & $50(78.1)$ & $22(68.8)$ \\
\hline - Black & $5(7.8)$ & $8(25.0)$ \\
\hline - Asian & $6(9.4)$ & $0(0.0)$ \\
\hline - Other & $3(4.7)$ & $2(6.3)$ \\
\hline Age (SD) & $64.8(13.6)$ & $53.3(19.0)$ \\
\hline Male & $34(53.1)$ & $19(59.4)$ \\
\hline \multicolumn{3}{|l|}{ Infection } \\
\hline - Catheter related & $4(4)$ & \\
\hline - CNS & $1(1.61)$ & \\
\hline - Endocarditis & $1(1.61)$ & \\
\hline - Intra-abdominal & $8(8)$ & \\
\hline - None & $2(3.23)$ & \\
\hline - Other & $3(4.84)$ & \\
\hline - Pneumonia & $21(33.87)$ & \\
\hline - Skin and soft tissue & $3(4.84)$ & \\
\hline - Unknown & $6(9.68)$ & \\
\hline - Urosepsis & $13(20.97)$ & \\
\hline \multicolumn{3}{|l|}{ Sepsis-related variables } \\
\hline Leukocyte count (per microliter), mean (SD) & $14.6(10.0)$ & \\
\hline Serum lactate $(\mathrm{mmol} / \mathrm{l})$, mean (SD) & $2.0(1.8)$ & \\
\hline SOFA score, mean (SD) & $7.4(3.5)$ & \\
\hline Mortality rate, $n(\%)$ & $11(17 \%)$ & \\
\hline
\end{tabular}

moving at a slower velocity than the column of erythrocytes [10-12]. This is similar to the definitions used in studies utilizing intravital microscopy. Thus, rolling leukocytes are identified as slow-moving leukocytes moving along the endothelial surface with temporary low-affinity bindings that are continuously overcome by the shear forces of the main blood flow, resulting in the characteristic rolling motion. Also, rolling leukocytes can detach from the endothelial cells and go back into the main blood 


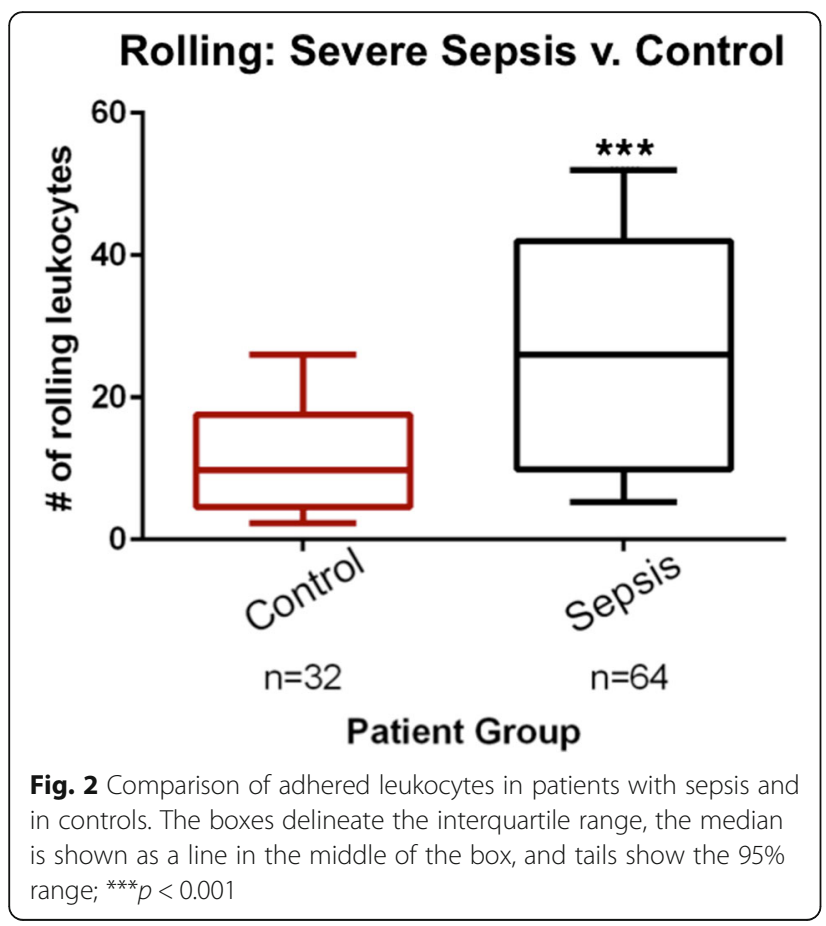

stream. We regarded all leukocytes moving slower, even stopping intermittently, or detaching back into the main bloodstream as rolling. In intravital studies leukocyte adherence is defined as leukocytes sticking to the endothelium for a given time; however the time often varies. Definitions vary between 1 and $60 \mathrm{~s}$ (even $10 \mathrm{~min}$ ), with $>30 \mathrm{~s}$ being the most common,

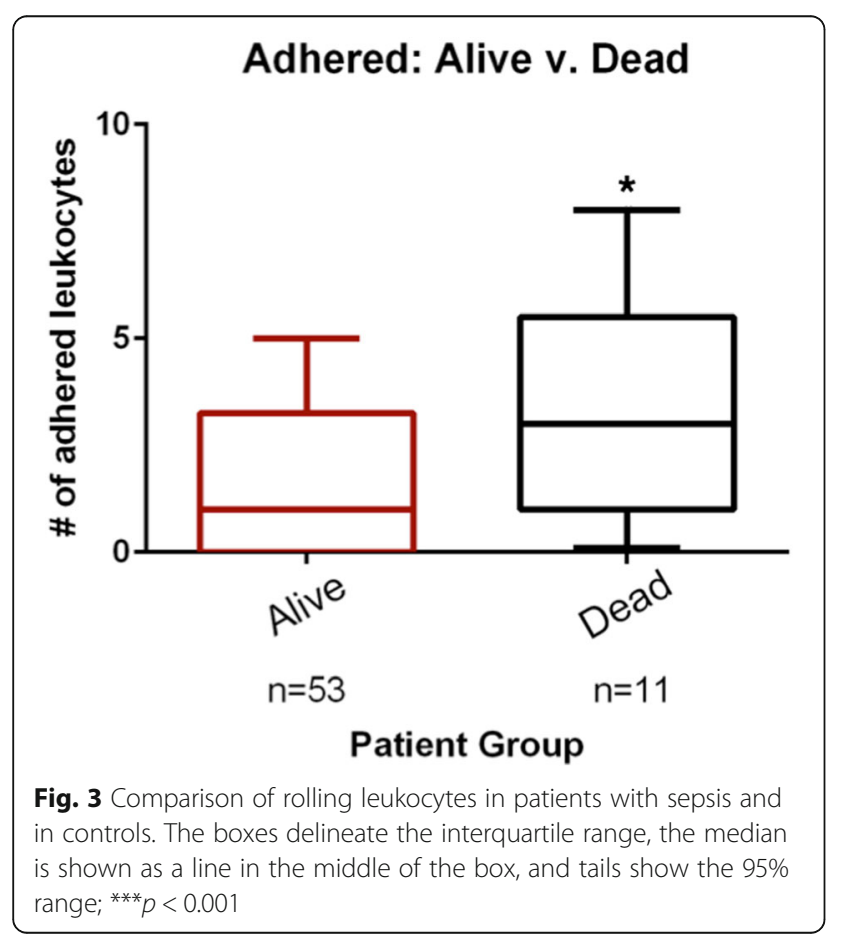

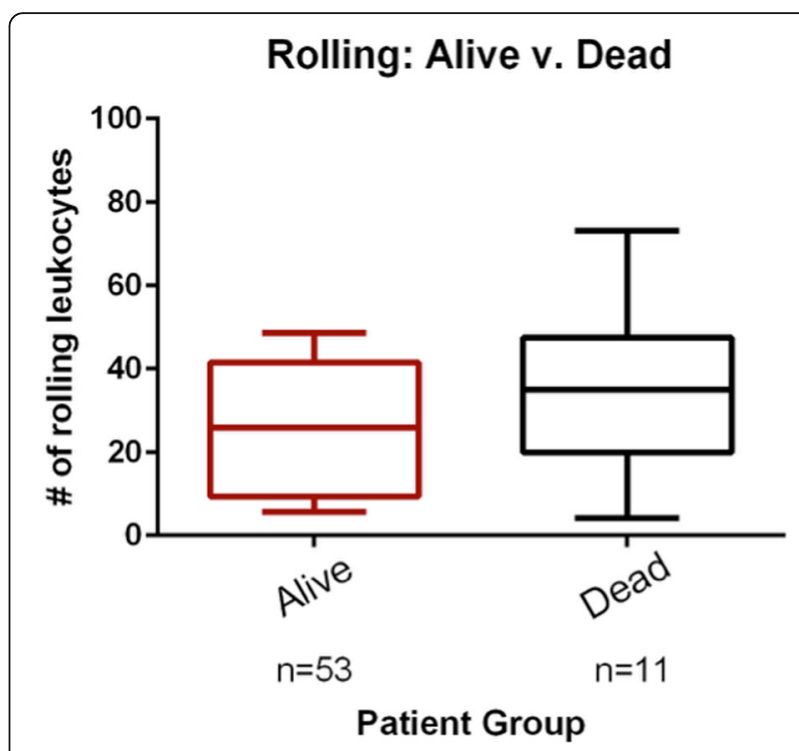

Fig. 4 Comparison of adhered leukocytes in survivors and non-survivors in the sepsis group. The boxes delineate the interquartile range, the median is shown as a line in the middle of the box, and tails show the $95 \%$ range; ${ }^{*} p<0.05$

which correlates to the time that a leukocyte needs to stay firmly adhered to initiate extravasation [2]. Observations in the human sublingual area of a single vessel segment over a long period of time are very difficult. We defined adhered leukocytes as leukocytes that started and remained in the same place on the endothelium throughout the video ( $3 \mathrm{~s}$ ).

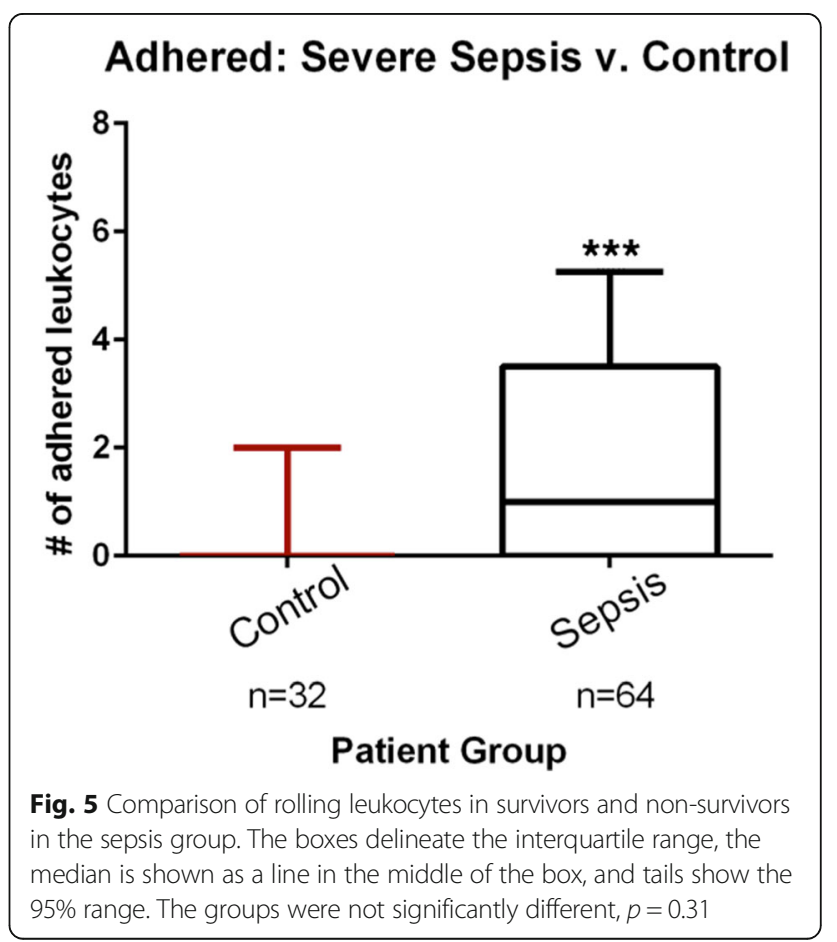


Previous studies with OPS imaging, the predecessor of SDF imaging, assessed sublingual leukocyte activation in humans during cardiopulmonary bypass and in humans exposed to high altitude $[1,10,11,20]$. Despite no alterations in microcirculatory flow velocity, a significant increase in activated (rolling) leukocytes observed sublingually during cardiopulmonary bypass along with significant correlation with CD18 expression (and systemic white blood cell (WBC) count) was seen. Only a moderate decrease in functional capillary density was seen. Thus, the authors found evidence of a systemic inflammatory reaction to cardiopulmonary bypass, which could be directly visualized sublingually independently of microcirculatory flow velocity [11]. This supports the hypothesis that the sublingual area can be used as a surrogate window to visualize systemic inflammatory reactions. In a study looking at the sublingual microcirculation during exposure to high altitude, there were no observed differences in leukocyte rolling or microcirculatory flow [10].

In an investigation similar to ours, Donati et al. quantified activated leukocytes along with measurements of glycocalyx degradation in critically ill patients and healthy control patients using SDF imaging [12]. They found significant correlation between glycocalyx degradation and number of rolling leukocytes, suggesting that leukocyte activation and endothelial injury may be assessed through the sublingual mucosa as an early diagnostic modality in sepsis [12]. Donati et al. compared the sublingual vascular glycocalyx between critically ill ICU patients and healthy volunteers. The perfused boundary region (PBR), which is proposed as a parameter inversely related to glycocalyx thickness, was increased in ICU patients compared to controls and most profound among ICU patients with sepsis. Donati et al. found a positive relationship between rolling leukocytes and PBR (presumed indicator of thinned glycocalyx) [12]. This adds evidence in support of the proposition that leukocyte activation and endothelial injury may be assessed through the sublingual mucosa in patients with systemic inflammation - in this case early septic shock.

Future directions include refinement, standardization, and/or automation of techniques to quantify the presence of rolling and adhered leukocytes. Alternative methods may utilize approaches used in intravital microscopy, e.g. measuring a rolling flux of leukocytes (count of rolling leukocytes crossing a designated perpendicular line) per time unit, or simply restrict the analysis to a fixed number of vessels. Another approach could be the subdividing of the FOV, so that investigators do not have to keep track of activated (rolling) leukocytes in the whole FOV $[10,11]$. Also, as the resolution of videomicroscopy imaging devices improves, visualization may be more accurate.

\section{Limitations of the study}

We lack an available automated technique for quantification of rolling and adhered leukocytes. We relied on manual identification of rolling and adhered leukocytes, which is prone to human error or imprecision, leaving us susceptible to misclassification bias. Another limitation is our convenience samples of patients and controls, leaving us prone to selection bias. In addition to that, we excluded videos if they did not conform to certain quality criteria.

A previous study showed that hemorrhage in rats reduced shear rate resulting in increased leukocyte-endothelial cell adhesion [21]. However, whether this is a physical phenomenon or a molecular one is not clearly delineated. We did not have hemodynamic data available at the time of image acquisition so we were unable to consider the influence of these measures on rolling and adhesion. We also did not stratify by vessel diameter, observing leukocytes primarily confined to post-capillary venules with a diameter greater than $20 \mu \mathrm{m}$; it is possible that discriminating by size or type of vessel may yield different numbers. Although less time consuming compared to the traditional manual analysis of microcirculatory flow parameters, leukocyte quantification is still quite time consuming - especially when counting in the whole field of view.

\section{Conclusions}

We demonstrated the physiologic expression of endothelial cell activation manifested by a significantly higher number of rolling and adhered leukocytes in patients with septic shock as compared to non-infected control patients. In the sepsis group there were more rolling and adhered leukocytes as compared to non-infected control patients. In the sepsis group, there were more adhered leukocytes in non-survivors compared to survivors. The sublingual mucosa may be a window to an important physiologic manifestation of leukocyte and endothelial cell activation in sepsis.

\section{Additional file}

Additional file 1: Videomicroscopy Movie of Adhered Leukocytes. (DOCX $42 \mathrm{~kb}$ )

\begin{abstract}
Abbreviations
ED: Emergency department; FDA: Food and Drug Administration; FOV: Field of view; ICU: Intensive care unit; LED: Light-emitting diode; OPS: Orthogonal polarization spectral; PBR: Perfused boundary region; ProCESS: Protocolized Care for Early Septic Shock; SD: Standard deviation; SDF: Sidestream dark field; SIRS: Systemic inflammatory response syndrome; WBC: White blood cell count
\end{abstract}

Acknowledgements

We thank the clinical staff and research staff from the participating hospitals who made this work possible. 


\section{Funding}

Supported by a grant NIH/NHLBI (R01 HL091757) and NIH/NIGMS (P50 GM076659) from the National Institutes of Health. BF was supported by a research grant from TrygFonden.

\section{Availability of data and materials}

The datasets used and/or analyzed during the current study are available from the corresponding author on reasonable request.

\section{Authors' contributions}

All authors contributed to study design. MF, PH, HW, DY, WA, JK, DA, and NS were responsible for patient enrollment and image acquisition. BF and MM reviewed and analyzed images. BF, MM, and NS performed statistical analysis and wrote first draft of the manuscript. BF and MM contributed equally to the manuscript. All authors made revisions to the manuscript and read and approved the final manuscript.

\section{Ethics approval and consent to participate}

The Beth Israel Deaconess Medical Center Committee for Clinical Investigations and each site's review board approved the design. Each subject or legal representative gave written informed consent.

\section{Consent for publication}

Not applicable.

\section{Competing interests}

NIS received funding from the National Institutes of Health; research funding from Cheetah Medical, Thermo-Fisher, Siemens, and Rapid Pathogen Screening. He was on an advisory board/consultant for Baxter and Cyon. The MicroScan equipment was provided by MicroVision Medical BV.

\section{Publisher's Note}

Springer Nature remains neutral with regard to jurisdictional claims in published maps and institutional affiliations.

\section{Author details}

'Department of Emergency Medicine, Beth Israel Deaconess Medical Center, Harvard Medical School, 1 Deaconess Road, CC2-W, Boston, MA 02215, USA. ${ }^{2}$ Research Center for Emergency Medicine, Aarhus University Hospital, Aarhus, Denmark. ${ }^{3}$ Department of Emergency Medicine, Massachusetts General Hospital, Harvard Medical School, Boston, MA, USA. ${ }^{4}$ Department of Emergency Medicine, Brigham and Women's Hospital, Harvard Medical School, Boston, MA, USA. ${ }^{5}$ Department of Emergency Medicine, University of Alabama at Birmingham, Birmingham, AL, USA. ${ }^{6}$ Department of Emergency Medicine, University of Pittsburgh, Pittsburgh, PA, USA. ${ }^{7}$ Center for Vascular Biology and Department of Medicine, Beth Israel Deaconess Medical Center, Harvard Medical School, Boston, MA, USA. ${ }^{8}$ Department of Critical Care Medicine, University of Pittsburgh, Pittsburgh, PA, USA.

Received: 23 March 2018 Accepted: 28 August 2018

Published online: 30 September 2018

\section{References}

1. Peters K, Unger RE, Brunner J, Kirkpatrick CJ. Molecular basis of endothelial dysfunction in sepsis. Cardiovasc Res. 2003;60(1):49-57.

2. Granger DN, Kubes P. The microcirculation and inflammation: modulation of leukocyte-endothelial cell adhesion. J Leukoc Biol. 1994;55(5):662-75.

3. Croner RS, Hoerer E, Kulu Y, Hackert T, Gebhard MM, Herfarth C, Klar E. Hepatic platelet and leukocyte adherence during endotoxemia. Critical care. 2006;10(1):R15

4. Farquhar I, Martin CM, Lam C, Potter R, Ellis CG, Sibbald WJ. Decreased capillary density in vivo in bowel mucosa of rats with normotensive sepsis. J Surg Res. 1996;61(1):190-6.

5. Al-Banna NA, Pavlovic D, Bac VH, Utpatel K, Janke E, Rippke JN, Borowiak M, Cerny V, Spassov A, Johnston B, et al. Acute administration of antibiotics modulates intestinal capillary perfusion and leukocyte adherence during experimental sepsis. Int J Antimicrob Agents. 2013:41(6):536-43.

6. De Backer D, Creteur J, Preiser JC, Dubois MJ, Vincent JL. Microvascular blood flow is altered in patients with sepsis. Am J Respir Crit Care Med. 2002;166(1):98-104.
7. Sakr Y, Dubois MJ, De Backer D, Creteur J, Vincent JL. Persistent microcirculatory alterations are associated with organ failure and death in patients with septic shock. Crit Care Med. 2004;32(9):1825-31.

8. Arnold RC, Dellinger RP, Parrillo JE, Chansky ME, Lotano VE, McCoy JV, Jones AE, Shapiro NI, Hollenberg SM, Trzeciak S. Discordance between microcirculatory alterations and arterial pressure in patients with hemodynamic instability. J Crit Care. 2012;27(5):e531.e1-7.

9. Ince C. Hemodynamic coherence and the rationale for monitoring the microcirculation. Crit Care. 2015;19(Suppl 3):S8.

10. Bauer A, Demetz F, Hoeper I, Thiel M, Chouker A, Christ F. Microvascular perfusion measured by orthogonal polarization spectral imaging is well maintained during exposure to high altitude in trained mountaineers. Eur J Med Res. 2008;13(12):568-75.

11. Bauer A, Kofler S, Thiel M, Eifert S, Christ F. Monitoring of the sublingual microcirculation in cardiac surgery using orthogonal polarization spectral imaging: preliminary results. Anesthesiology. 2007;107(6):939-45.

12. Donati A, Damiani E, Domizi R, Romano R, Adrario E, Pelaia P, Ince C, Singer M. Alteration of the sublingual microvascular glycocalyx in critically ill patients. Microvasc Res. 2013;90:86-9.

13. Yealy DM, Kellum JA, Huang DT, Barnato AE, Weissfeld LA, Pike F, Terndrup T, Wang HE, Hou PC, LoVecchio F, et al. A randomized trial of protocolbased care for early septic shock. N Engl J Med. 2014;370(18):1683-93.

14. Levy MM, Fink MP, Marshall JC, Abraham E, Angus D, Cook D, Cohen J, Opal SM, Vincent JL, Ramsay G. 2001 SCCM/ESICM/ACCP/ATS/SIS international Sepsis definitions conference. Intensive Care Med. 2003;29(4):530-8.

15. De Backer D, Hollenberg S, Boerma C, Goedhart P, Buchele G, OspinaTascon G, Dobbe I, Ince C. How to evaluate the microcirculation: report of a round table conference. Crit Care. 2007;11(5):R101.

16. Massey MJ, Larochelle E, Najarro G, Karmacharla A, Arnold R, Trzeciak S, Angus DC, Shapiro NI. The microcirculation image quality score: development and preliminary evaluation of a proposed approach to grading quality of image acquisition for bedside videomicroscopy. J Crit Care. 2013;28(6):913-7.

17. Alahi A, Ortiz R, Vandergheynst P. FREAK: fast retina Keypoint. In: IEEE Conference on Computer Vision and Pattern Recognition. Providence: leee; 2012.

18. Frangi A, Niessen W, Vincken K, Viergever M. Multiscale vessel enhancement filtering. In: Wells W, Colchester A, Delp S, editors. Medical image computing and computer-assisted interventation - MICCAI'98 vol 1496. Berlin Heidelberg: Springer; 1998. p. 130-7.

19. Zuiderveld K. Contrast limited adaptive histogram equalization. In: Paul SH, editor. Graphics gems IV. Chicago: Academic Press Professional, Inc; 1994. p. 474-85.

20. O'Neil MP, Fleming JC, Badhwar A, Guo LR. Pulsatile versus nonpulsatile flow during cardiopulmonary bypass: microcirculatory and systemic effects. Ann Thorac Surg. 2012;94(6):2046-53.

21. Nakagawa NK, Nogueira RA, Correia CJ, Shiwa SR, Costa Cruz JW, Poli de Figueiredo LF, Rocha ESM, Sannomiya P. Leukocyte-endothelium interactions after hemorrhagic shock/reperfusion and cecal ligation/ puncture: an intravital microscopic study in rat mesentery. Shock. 2006; 26(2):180-6.

Ready to submit your research? Choose BMC and benefit from:

- fast, convenient online submission

- thorough peer review by experienced researchers in your field

- rapid publication on acceptance

- support for research data, including large and complex data types

- gold Open Access which fosters wider collaboration and increased citations

- maximum visibility for your research: over $100 \mathrm{M}$ website views per year

At $\mathrm{BMC}$, research is always in progress.

Learn more biomedcentral.com/submissions 